\title{
CONSTRUÇÃO E VALIDAÇÃO DE APLICATIVO MÓVEL PARA AUTOCUIDADO NO CONTROLE DA PRESSÃO ARTERIAL DE GESTANTES
}

\author{
CONSTRUCTION AND VALIDATION OF A MOBILE APPLICATION FOR SELF-CARE IN \\ THE CONTROL OF BLOOD PRESSURE IN PREGNANT WOMEN
}

\section{Raylla Araújo Bezerra ${ }^{1} *$ Gabriela Silva Esteves de Hollanda $^{2} *$ Gabrielle Santiago Ribeiro $^{3} *$ Erivando de Sena Ramos $^{4} *$ Alexia Maria da Costa Patrício ${ }^{5} *$ Lydia Vieira Freitas dos Santos $^{6}$}

\section{RESUMO}

Objetivo: elaborar e validar um aplicativo em plataforma móvel direcionado para o autocuidado de gestantes no controle da pressão arterial. Método: estudo metodológico. Realizada a validação quanto ao conteúdo e aparência, junto à equipe multidisciplinar com especialistas e a validação junto a representantes do público-alvo. Resultados: o aplicativo "GestHiper" teve como etapas de sua construção o levantamento de dados, a construção dos tópicos, navegabilidade e programação do aplicativo. Quanto à validação, este obteve um Índice de Validação de Conteúdo global de 0,95, sendo considerado validado. Mediante as recomendações dos especialistas e gestantes, o aplicativo passou por ajustes a fim de tornar-se mais eficaz. Conclusão: acredita-se que as informações e ferramentas contidas no aplicativo móvel contribuirão de forma significante para a aquisição de conhecimento e monitoramento efetivo da pressão arterial das gestantes que dele se utilizar.

Keywords: Educational Technology; Mobile Applications; Pregnancy; Arterial Pressure; Self Care.

\begin{abstract}
Objective: To develop and validate an application on a mobile platform aimed at self-care for pregnant women without blood pressure control. Method: methodological study. Validation was carried out as to content and requirement, with the multidisciplinary team with experts and validation with representatives of the target audience. Results: the "GestHiper" application had as its construction stages the data collection, the construction of the old ones, navigability and application programming. As for validation, this obtained an overall Content Validation Index of 0.95 , being considered validated. Upon the recommendations of experts and pregnant women, the application underwent adjustments in order to become more effective. Conclusion: it is believed that the information and tools contained in the mobileapplication will significantly contribute to the acquisition of knowledge and effectivemonitoring of blood pressure in pregnant women who use it.
\end{abstract} Keywords: Educational Technology; Mobile Applications; Pregnancy; Arterial Pressure; Self Care.

\footnotetext{
${ }^{1}$ Doutoranda pela Universidade Federal do Ceará. Fortaleza, Ceará, Brasil. ORCID: https://orcid.org/0000-0002-2781-7817

${ }^{2}$ Mestra pela Universidade Federal da Paraíba. Recife, Pernambuco, Brasil. ORCID: https://orcid.org/0000-0002-3612-692X

${ }^{3}$ Graduanda em Enfermagem pela Universidade da Integração Internacional da Lusofonia Afro-Brasileira. Fortaleza, Ceará, Brasil. ORCID: https://orcid.org/0000-0001-8606-626X

${ }^{4}$ Mestre em Sociobiodiversidade e Tecnologias Sustentáveis pela Universidade da Integração Internacional da Lusofonia AfroBrasileira. Redenção, Ceará- Brasil. ORCID: https://orcid.org/0000-0002-1079-9704

${ }^{5}$ Graduanda em enfermagem pela Universidade da Integração Internacional da Lusofonia Afro-Brasileira. Itapipoca, Ceará, Brasil ORCID: https://orcid.org/0000-0002-8505-3261

${ }^{6}$ Doutora. Professora adjunta da Universidade da Integração Internacional da Lusofonia Afro-Brasileira. Fortaleza, Ceará, Brasil. ORCID: https://orcid.org/0000-0003-4277-7486 


\section{INTRODUÇÃO}

A gestação compreende um evento fisiológico para as mulheres. Nesse período, ocorrem mudanças em nível físico, psicológico e familiar. A gravidez faz parte do desenvolvimento e carece de reestruturação, equilíbrio e reajuste em certas circunstâncias como em agravos à saúde do binômio materno-fetal ${ }^{(1)}$.

Dentre as complicações reconhecidas na literatura, as mais citadas são a piora do quadro hipertensivo, pré-eclâmpsia, restrição do crescimento fetal, parto prematuro, descolamento prematuro da placenta e óbito fetal $^{(2)}$. A identificação adequada e prévia de gestantes com riscos e que podem evoluir a um prognóstico desfavorável necessita ocorrer no pré-natal por profissionais qualificados, pois podem evoluir para mortalidade materna ${ }^{(3)}$.

As principais causas de morte nas mulheres foram relacionadas aos agravos da gravidez e comorbidades preexistentes, sendo estas as hemorragias, Hipertensão Arterial Sistêmica (HAS), infecções e causas indiretas $^{(4)}$. Logo, gestantes com alto risco para a SHG que desconhecem o agravamento e as ações para prevenção e/ou controle dos fatores de risco podem ter comportamentos inapropriados de autocuidado ${ }^{(5)}$.

Desse modo, faz-se premente a adoção de práticas educativas para possibilitar o https://doi.org/10.31011/reaid-2021-v.95-n.35-art.1186 autocuidado das gestantes frente à sua enfermidade e o manejo da SHG, bem como o investimento em tecnologias que possam proporcionar maior autonomia à gestante no controle da pressão arterial (PA) durante esse período.

A partir do exposto e ressaltando a importância da inovação tecnológica e da elaboração de tecnologias educativas que colaborem com as atividades diárias de controle da PA durante a gestação, surgiu o interesse no desenvolvimento de um aplicativo para dispositivo móvel (GestHiper), destinado para uso da gestante normotensa e hipertensa, almejando dar-lhe suporte na avaliação de seu nível pressórico rotineiramente e apoio ao autocuidado com informações para adesão a práticas saudáveis de alimentação e exercícios físicos, bem como para gestantes com hipertensão arterial crônica ou com SHG instalada possam fazer o uso do aplicativo para monitorar a PA.

Diante disso, o estudo teve como objetivo elaborar e validar um aplicativo em plataforma móvel direcionado para o autocuidado de gestantes normotensas e hipertensas no controle da pressão arterial.

\section{MÉTODO}

\section{Tipo de estudo}

Trata-se de um estudo metodológico, de produção tecnológica, realizado no período entre outubro de 2016 a fevereiro de 2018. E 
para a realização do presente estudo foi utilizado o modelo de Falkembach, sendo executadas 4 das suas 5 etapas.

\section{Etapa 1- Análise e Planejamento}

$\mathrm{Na}$ primeira fase foi definido o tema, considerado os recursos disponíveis, definido o objetivo da aplicação, público-alvo, a utilização do produto, quando, onde, para que, e os resultados esperados com o seu uso ${ }^{(6)}$. Foi realizado um amplo resgate da literatura existente referente à SHG e extraídas as principais condutas para prevenção, controle e manejo da doença a partir de uma revisão integrativa da literatura.

Além da consulta à literatura, foi realizada uma roda de conversa com um grupo educativo de gestantes com a finalidade de conhecer melhor as principais dúvidas e dificuldades dessas mulheres no período gestacional relacionados aos transtornos hipertensivos.

\section{Etapa 2- Modelagem}

$\mathrm{Na}$ segunda fase foram criados três modelos: o conceitual, de navegação e de interface. $\mathrm{O}$ modelo conceitual refere-se ao conteúdo da aplicação e ao modo como esse conteúdo está disponibilizado ao usuário. Já o modelo de navegação define como são os links entre as estruturas através do uso de menus, índices, roteiros guiados. E o modelo de interface deve estar em acordo com o modelo conceitual e de navegação ${ }^{(6)}$.

\section{Etapa 3- Implementação}

Na terceira fase, a implementação, que trata da construção da primeira versão do aplicativo que inclui a criação das mídias, incluindo imagens, animações e textos houve a participação de um profissional da Tecnologia da Informação (TI) especializado em construção de aplicativos.

\section{Etapa 4- Avaliação e Manutenção}

Nesta fase a avaliação foi realizada por especialistas das áreas de Obstetrícia/PréNatal, Saúde Cardiovascular, Nutrição, Educação física e Tecnologia da Informação, com a finalidade de garantir a credibilidade da tecnologia em saúde ${ }^{(7)}$. 
Figura 1- Fluxograma das fases seguidas no desenvolvimento do aplicativo móvel a partir do modelo de Falkembach.

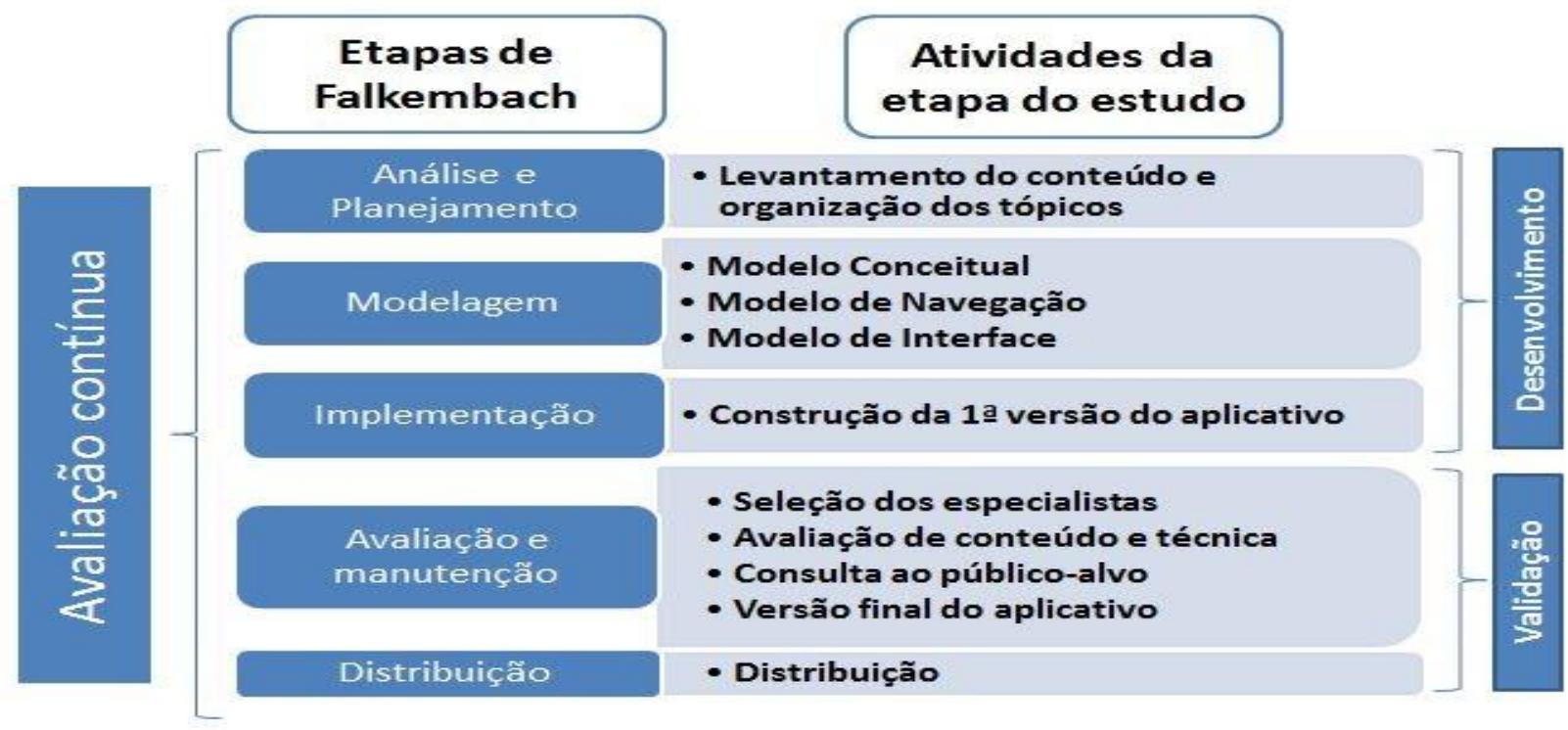

Fonte: A partir do modelo de Falkembach.

\section{População e definição da amostra}

Para a validação do aplicativo móvel, os especialistas foram selecionados através do tipo de amostragem não probabilística e por conveniência. Quanto ao número de especialistas, a presente pesquisa optou por seguir as recomendações, onde o número de especialistas seja maior que seis e que a quantidade seja em número ímpar ${ }^{(8)}$. Dessa forma, foram recrutados sete especialistas de cada área de interesse, de acordo com a avaliação do currículo lattes, sendo sete especialistas obstetras, sete enfermeiros, sete nutricionistas, sete cardiovasculares e sete educadores físicos. $\mathrm{O}$ instrumento foi adaptado dos instrumentos utilizados em estudos de validação ${ }^{(7,9)}$.
Para consulta ao público-alvo, foram captadas gestantes, de acordo com os critérios de inclusão, que são: gravidez comprovada; gestantes alfabetizadas; ter iniciado o prénatal; manifestar interesse em participar da pesquisa; tempo necessário disponível para participação. Dentre estas mulheres, foram captadas onze gestantes, sendo este um número aceitável de avaliadores para estudos como este ${ }^{(8)}$.

\section{Coleta de dados}

Foram enviados para os especialistas, via e-mail, uma Carta-convite, contendo a proposta da pesquisa e o Termo de Consentimento Livre e Esclarecido (TCLE). A partir do aceite de participação e assinatura do TCLE o instrumento de avaliação do aplicativo foi enviado via ferramenta da 
Google, o google forms. O instrumento foi dividido em duas partes: a primeira contém itens de caracterização dos sujeitos; e a segunda traz os itens avaliativos do aplicativo.

As respostas às questões foram apresentadas sob a forma de escala tipo Likert, onde: 1. Inadequado; 2. Parcialmente adequado; 3. Adequado; 4. Totalmente adequado e NA (não se aplica) e divididas em três subcategorias: Objetivos, Relevância e conteúdo.

Já os especialistas em Tecnologia da Informação avaliaram a técnica do aplicativo de acordo com os quesitos: funcionalidade, usabilidade e eficiência do aplicativo $^{(7)}$. Os quesitos objetivam avaliar a adequabilidade do sistema para o usuário e seus resultados sistema $^{(10)}$.

A coleta com o público-alvo foi realizada através de uma sessão no dia da consulta de pré-natal para orientação sobre a navegação no aplicativo. Em seguida, ocorreu uma navegação conjunta. Após a navegação, foi solicitado que as gestantes lessem e assinassem o TCLE e fizessem a avaliação do aplicativo. $\mathrm{O}$ instrumento direcionado ao público-alvo foi adaptado e avaliavam objetivos, organização, estilo da escrita, aparência e motivação em utilizar o aplicativo. como para a engenharia de construção do

Para a validação da plataforma móvel, foram utilizadas duas estratégias de validação dos itens. A primeira, adaptada, que considera validado o item que recebe a classificação 4 "Totalmente Adequado" por, pelo menos, metade dos especialistas, e que não recebe a classificação 1 "Totalmente Inadequado". O item também é considerado validado quando recebe a classificação 3 "Parcialmente Adequado" ou 2 "Inadequado", mas apresenta sugestões de melhoria e estas são implementadas $^{(7)}$.

Além desses quesitos, os itens deveriam apresentar um Índice de Validação de Conteúdo (IVC) maior ou igual a 0,78. Verificando se os conceitos estão representados de modo adequado, bem como se os itens ou textos do instrumento estão em concordância entre as opiniões dos especialistas $^{(11)}$. Calculando a proporção ou porcentagem de especialistas que estão em concordância em certos pontos do instrumento e de seus itens, este permite analisar cada item de forma individual e em seguida o instrumento de forma geral $^{(12)}$.

A fim de avaliar a confiabilidade e validade do instrumento para avaliação do aplicativo foram aplicados testes estatísticos, a saber, Índice de Kappa generalizado, Coeficiente de correlação intraclasse e Coeficiente Alfa de Cronbach. Para uma certificação a mais da avaliação dos profissionais a respeito do aplicativo para https://doi.org/10.31011/reaid-2021-v.95-n.35-art.1186 Rev Enferm Atual In Derme v. 95, n. 35, 2021 e-021124 
auxílio das gestantes, foi aplicado os testes Kappa generalizado e o coeficiente de correlação intraclasse (CCI), objetivando verificar a concordância entre as respostas dos profissionais, e também o alfa de cronbach para analisar a confiabilidade do aplicativo.

Posteriormente à avaliação de conteúdo, técnica e à consulta ao público-alvo foi elaborada a versão final do aplicativo levando em consideração as sugestões preenchidas nos formulários dos especialistas aprimorar o estudo.

O projeto foi submetido à avaliação do Comitê de Ética em pesquisa da Universidade da Integração Internacional da Lusofonia Afro-Brasileira e aprovado em 14 de dezembro de 2017, sob parecer $n^{\circ} 2.438 .434$. Foi garantido sigilo sobre todas as informações coletadas, assegurando o anonimato dos participantes, seguindo as normas da Resolução $\mathrm{N}^{\mathrm{o}} \quad 466 / 2012$ do Conselho Nacional de Saúde do Ministério da Saúde para pesquisa com seres humanos.

\section{RESULTADOS}

\section{Fase 1 - construção do aplicativo móvel}

\section{Levantamento de dados}

A etapa inicial da construção do aplicativo móvel consistiu no levantamento de conteúdo com relação ao autocuidado da e do público-alvo para contemplar e

pressão arterial, para que fosse possível organizá-lo em módulos.

Destacam-se como materiais fundamentalmente utilizados: Tratado da Fisiologia Médica, dos autores Guyton e Hall; Diretriz brasileira de hipertensão arterial, da Sociedade Brasileira de Cardiologia; Caderno de Atenção Básica: Atenção ao pré-natal de alto risco, do Ministério da Saúde; Orientações Nutricionais: da gestação à primeira infância, do Senado Federal; Alimentação e nutrição na gravidez. Programa Nacional para a Promoção da Alimentação Saudável. E ainda foram utilizados 2 artigos que tratavam acerca da prática do exercício físico na gestação e da utilização de plantas medicinais potencialmente nocivas, respectivamente.

Foram utilizados 2 gifs e 8 figuras, extraídas de um banco de imagens gratuito da Google. Os gifs foram selecionados com a finalidade de representarem, de forma mais real, os mecanismos explicados.

A partir do levantamento de dados, os achados de maior relevância no conteúdo, foram estabelecidos como os principais tópicos que deveriam constituir o aplicativo móvel, cujo nome comercial foi definido GestHiper.

\section{Construção dos tópicos}

Inicialmente, ao abrir o aplicativo, uma tela de boas-vindas é apresentada https://doi.org/10.31011/reaid-2021-v.95-n.35-art.1186 Rev Enferm Atual In Derme v. 95, n. 35, 2021 e-021124 
juntamente com informações sobre seus objetivos e ferramentas, através de um breve texto de caráter interativo.

Posteriormente na tela introdutória encontra-se o cadastro para ser preenchido com as principais informações relacionadas à identificação e dados da gestação. O único campo obrigatório do cadastro é o da identificação, entretanto, se a mulher fornecer a data da sua última menstruação o aplicativo calcula e apresenta sua data provável de parto e idade gestacional.

Figura 2- Tela dos tópicos e "Você precisa saber" do aplicativo

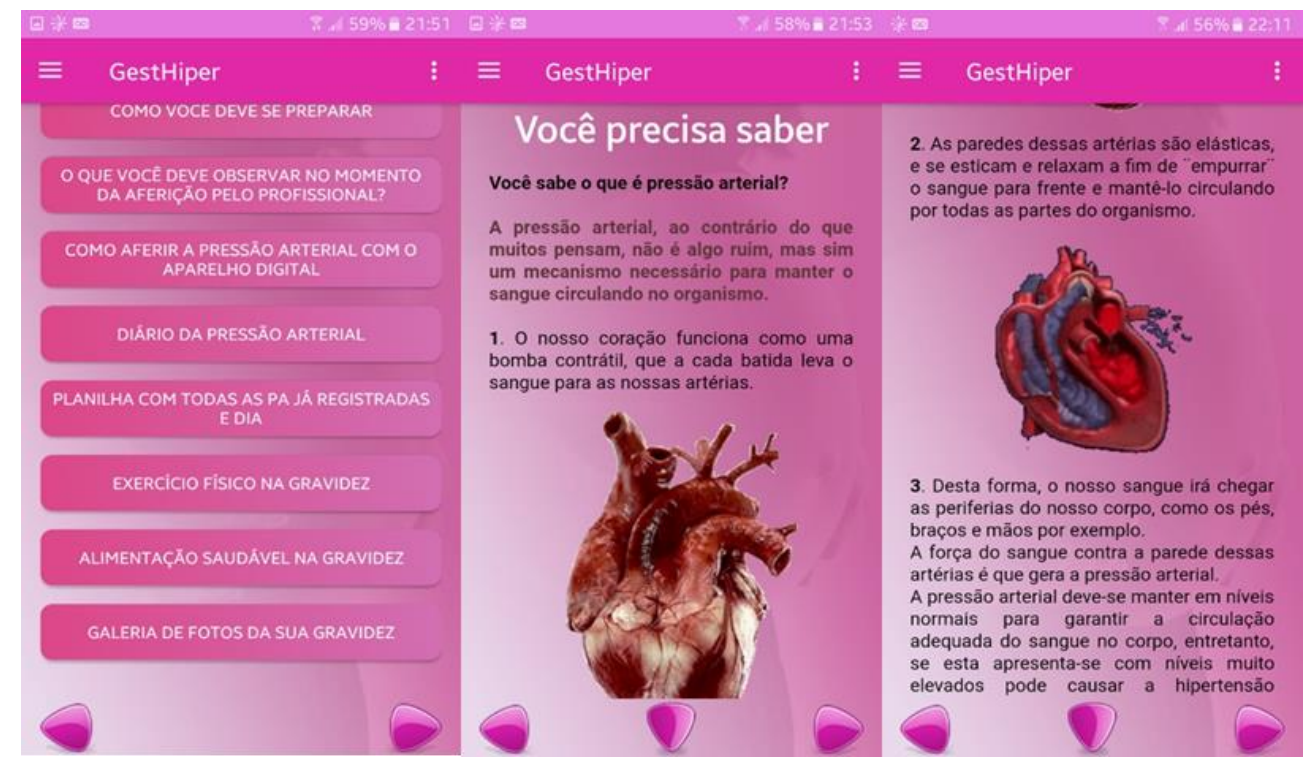

Fonte: Aplicativo GestHiper (2018).

Quanto à construção dos tópicos, buscou-se organizar o conteúdo do aplicativo em tópicos independentes para que a plataforma móvel se apresentasse de forma didática e de fácil aprendizagem, facilitando assim o acesso da gestante em curto espaço de tempo, bem como oferecendo a autonomia de acessar o tópico que desejar na sequência que considerar favorável.

Tendo sido a gestante apresentada à temática da plataforma móvel, existe ainda a necessidade de explanar o conceito e os aspectos que se relacionam a pressão arterial, Desta forma, foi introduzido um tópico denominado "Você precisa saber", o qual apresenta o conceito e aspectos do que seria pressão arterial e outro tópico denominado “ $\mathrm{O}$ que é hipertensão e síndrome hipertensiva gestacional" que abordam as patologias hipertensivas.

No mesmo tópico "Você precisa saber" foram utilizados gifs da internet, com 
animação, de corações realizando sístole e diástole e vasos ligados a este órgão, demonstrando de forma didática a fisiologia do sistema circulatório, representando assim como a pressão arterial atua nos vasos. Optou-se por utilizar gifs com imagens do órgão real e o som dos batimentos cardíacos, para proporcionar uma explicação mais realista para as gestantes, evitando assim explanações abstratas de difícil compreensão.

No tópico seguinte "O que você deve saber antes de aferir sua pressão arterial" foi introduzido um texto que relata a importância de se preparar adequadamente para a aferição da pressão arterial. No tópico "O que você deve observar no momento da aferição pelo profissional" foram reportadas informações do que deveria ser analisado pela gestante quanto à prática do profissional no momento da aferição.

Optou-se por abordar também o uso do aparelho digital para aferição, incluindo assim um tópico que trazia o passo a passo do manuseio deste aparelho. A importância deste tópico se dá no empoderamento que a gestante pode adquirir de aferir sua pressão arterial em seu ambiente domiciliar, uma vez que disponha deste equipamento e não tenha como ter acesso imediato a um profissional que realize a aferição com o aparelho tradicional.

Perante a necessidade de se apresentar um tópico diferencial para o aplicativo, para que este despertasse ainda mais o interesse do público-alvo, construiu-se o tópico “Diário da pressão arterial". A função central é permitir o cadastro de todos os valores aferidos no decorrer da gestação; a cada valor registrado o aplicativo apresenta um feedback do que significa aquele valor, se está dentro dos padrões de normalidade ou não, apresentando também informações de como controlar a P.A. O diário de P. A. também possui a função de arquivo, salvando todos os valores registrados em uma planilha, que oferece o benefício de apresentar ao médico ou enfermeiro da gestante em cada consulta prénatal, oferecendo um acompanhamento mais preciso a esta mulher. 
Figura 3- Tela "Diário da pressão arterial" do aplicativo.

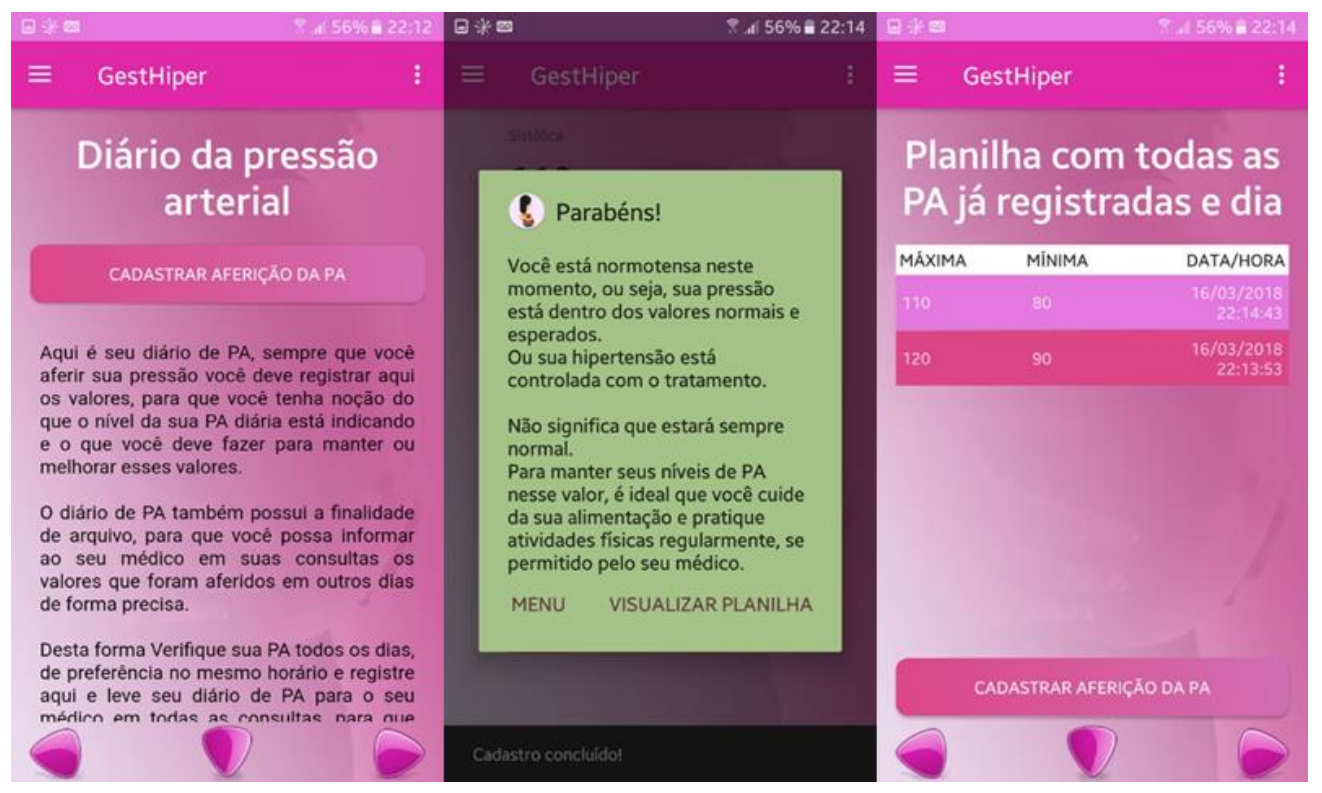

Fonte: Aplicativo GestHiper (2018).

No tópico "Exercício físico na gravidez" foram inseridas informações acerca dos benefícios do exercício físico regular durante a gravidez, incluindo também as atividades apropriadas e inapropriadas, bem como o tempo de duração adequado das atividades. Deixou-se claramente expressa a necessidade de uma avaliação com o médico antes de iniciar os exercícios e da instrução de um profissional de Educação Física para as atividades.

Com a finalidade de promover o melhor controle da PA na gravidez, construiuse o tópico "Alimentação saudável na gravidez" que buscou apresentar os nutrientes que devem ser priorizados neste período, trazendo exemplos de alimentos que possuem estes nutrientes, informações de quais alimentos evitar e o risco de ingerir alimentos crus ou malcozidos.

O último tópico do aplicativo trouxe um espaço interativo que permite que a usuária retire uma foto ou adquira da sua própria galeria, coloque legenda e salve na galeria de fotos do aplicativo, podendo ser exibidas individualmente ou em forma de slideshow. A finalidade do espaço interativo é permitir que a gestante construa um local individual e personalizado.

Foram utilizados 2 gifs e 8 figuras, extraídas de um banco de imagens gratuito da Google. Os gifs foram selecionados com a finalidade de representarem, de forma mais real, os mecanismos explicados. Quanto às figuras, buscou-se representar as mulheres 
grávidas em vários momentos diferentes, para possibilitar que estas se visualizassem realizando as atividades, ou sentindo-se bem por utilizarem o aplicativo e melhorar o autocuidado da PA.

\section{Navegabilidade}

O aplicativo "Gestante: Autocuidado da pressão arterial" foi desenvolvido nativamente para uso em dispositivos móveis que utilizem a plataforma Android compatível com o sistema operacional Android versão 4.0.3 (API 15) ou superiores até versão 8.0.0 (API 26). Para o acesso a plataforma foi disponibilizada um link que permitia o download do aplicativo no smartphone para que fosse realizado a avaliação. Quanto à navegabilidade, o utilizado do aplicativo poderia clicar no tópico que desejasse visualizar para ir diretamente a este ou navegar de tópico em tópico na sequência disponível.

O aplicativo foi projetado seguindo padrões de desenvolvimento de software. Durante a fase de preparação do ambiente de desenvolvimento foram utilizados os recursos da IDE Android Studio versão 3.0.0 instalado em um computador desktop com sistema operacional Windows 10 Professional 64Bits com suporte à tecnologia de virtualização Intel Virtualization Technology (VT-x). A linguagem de programação utilizada durante toda fase de desenvolvimento foi o Java versão 8 utilizando recursos da JDK versão 1.8.0_141.

$\mathrm{Na}$ fase de codificação com base nos requisitos fornecidos conforme projeto, foram aplicadas boas práticas de programação incluindo o padrão MVP (Model View Presenter) para Android.

$\mathrm{Na}$ fase de testes do aplicativo foram utilizados dispositivos virtuais através da ferramenta AVD da IDE Android Studio. Após compilação do código fonte foi gerado um arquivo com extensão APK(Android Package) em formato padrão para posterior publicação no Google Play Store.

\section{Fase 2 - validação do aplicativo móvel}

O aplicativo foi construído e posteriormente validado por especialistas da área da saúde, das seguintes áreas: Obstetrícia, Saúde cardiovascular, Educação Física, Nutrição e Tecnologia da Informação, bem como por representantes do público-alvo, composto por gestantes, seguindo as etapas anteriormente mencionadas.

No tópico "Alimentação saudável na gravidez", foi sugerida por parte do profissional nutricionista uma orientação mais clara acerca do consumo moderado de carboidratos, consumo de fibras e o controle do consumo de sal.

No tópico "Como verificar em casa e saber se está normal?", foi sugerida 
orientação com relação à periodicidade das aferições e a necessidade de acompanhamento ambulatorial simultâneo, bem como atualização dos valores normais da pressão arterial.

A amostra de representantes do público-alvo avaliou o aplicativo como bem organizado, linguagem compreensível, imagens simples, esclarecedor e educativo.

Na Tabela 1, são apresentados os resultados dos testes de confiança e validade para os profissionais da saúde, TI e também para o questionário aplicado as gestantes.

Tabela 1 - Análise de confiabilidade e validade na avaliação de profissionais de saúde, Tecnologia da informação (TI) e público alvo. Redenção, CE, Brasil, 2018. (n=46).

\begin{tabular}{llllllc}
\hline Área & $\mathbf{n}$ & Kappa & Valor $\mathbf{p}$ & $\mathbf{C C I}$ & Valor p & Alfa de Cronbach \\
\hline Área da saúde & 28 & 0,126 & $<0,001$ & 0,107 & $<0,001$ & 0,83 \\
\hline $\begin{array}{l}\text { Tecnologia da } \\
\text { informação }\end{array}$ & 7 & $-0,055$ & 0,207 & $-0,041$ & 0,713 & 0,876 \\
\hline Público alvo & 11 & $-0,0001$ & 0,997 & $-0,012$ & 0,589 & 0,748 \\
\hline
\end{tabular}

Fonte: Os autores.

Como pode ser visto na Tabela 1 , o índice de Kappa quanto à avaliação dos profissionais da área da saúde é 0,126, e o valor p associado é <0,001 que é menor que 0,05, portanto, ao nível de $95 \%$ de confiança pode-se dizer que existe concordância entre as avaliações dos profissionais da área da saúde, não sendo puramente aleatória. Já o índice de correlação intraclasse foi 0,107 , com valor $p$ também <0,001, indicando que existe correlação diferente de 0 . Ainda com relação à avaliação dos profissionais de saúde o alfa de Cronbach, que indica a confiabilidade do estudo é 0,83 , que é considerado um ótimo resultado, pois indica que a avaliação dos profissionais é muito consistente.
Na tabela 1 é visto também que não houve índices significativos para a área da tecnologia da informação e para as gestantes, no entanto, o valor do alfa de Cronbach mostrou-se excelente, principalmente para os profissionais da tecnologia da informação, que foi de 0,876 , indicando que no geral as respostas foram semelhantes e consistentes.

\section{DISCUSSÃO}

Os aplicativos móveis oferecem vantagens, seja na liberdade de movimento e portabilidade que conferem, quanto no nível de investimento mais racional que ensejam. Estudos nacionais e internacionais consistentemente sugerem que os aplicativos 
móveis serão cada vez mais utilizados por profissionais de saúde para tomadas de decisões ágeis e de melhor qualidade no cuidado prestado nos ambientes clínicos ${ }^{(13,14)}$.

Desta forma, o aplicativo construído para esse estudo é destinado à área da saúde com enfoque no autocuidado da pressão arterial por gestantes, e para sua construção foram priorizados os seguintes aspectos: exposição de temas pertinentes relacionados à Síndrome Hipertensiva Gestacional (SHG) e em linguagem acessível, a possibilidade de inserir e armazenar dados e fotos no aplicativo, inserir os valores pressóricos gerando telas explicativas, entre outros ${ }^{(15)}$.

A avaliação positiva do aplicativo tanto em relação à sua estrutura quanto em relação à sua facilidade de utilização, demonstra que a incorporação de novas tecnologias na avaliação da pressão arterial em gestantes pode ser uma realidade para a promoção do autocuidado.

Uma revisão de literatura sobre os processos de validação de instrumentos verificou a existência de diferentes métodos para quantificar o grau de concordância entre os especialistas e destacou a utilização do IVC, respaldando o método utilizado pelo presente estudo na análise dos dados referentes à validação de conteúdo e aparência pelos especialistas ${ }^{(16)}$. O aplicativo foi validado com IVC global de 0,95 , o que indica um ótimo grau de concordância entre os avaliadores sobre os aspectos que foram considerados.

Em relação à construção, a plataforma utilizada para o desenvolvimento do aplicativo é gratuita, amplamente disponível e permite a atualização dos dados com facilidade, mesmo por pessoas sem conhecimento de programação em sistemas de informação. Estas plataformas baseadas na web estão suficientemente desenvolvidas e seguras para uso na pesquisa e assistência em saúde, como verificado por estudos prévios $^{(14)}$.

O aplicativo foi projetado pela autora priorizando funcionalidade para o autocuidado das gestantes, mas dando a devida relevância a apresentação gráfica, objetivando simplicidade estética sem perda de conteúdo. A navegação do aplicativo acontece por menus que organizam o conteúdo de forma didática e de fácil acesso, como salientado em estudos recentes ${ }^{(17)}$.

Quanto ao conteúdo e funcionalidade do aplicativo, foram sugeridas algumas alterações pelos juízes, de modo que a linguagem utilizada deve almejar simplicidade e objetividade, evitando rebuscamento e excesso de informações de cunho não prático, ainda que, permitindo o aprofundamento sobre os tópicos através de links direcionados a referências disponíveis 
na internet. Além disso, o uso de recursos didáticos, como figuras, tabelas ou vídeos, torna mais simples o entendimento de conceitos complexos em curto período de tempo ${ }^{(17)}$.

É recomendável o uso de alimentos com baixa densidade calórica e baixo teor de gorduras saturadas, colesterol e gordura total, além do consumo de uma dieta rica em frutas e vegetais. Isso porque tem impacto importante na redução da PA, além de reduzir biomarcadores de risco cardiovascular. Entretanto, seguir uma alimentação com esse padrão é difícil em decorrência da facilidade de aquisição de alimentos de preparo rápido, que geralmente vêm acompanhados de alto valor calórico $^{(18)}$.

Considerando que a única forma de diagnosticar e controlar a hipertensão arterial são aferindo a pressão arterial (PA), sabe-se que há significativas diferenças entre a medida casual (valores obtidos por medidas no consultório) e a medida no domicílio, os métodos que se utilizam de aparelhos automáticos afastam diversos fatores que interferem nessa medida ${ }^{(18)}$.

A participação dos especialistas foi extremamente importante para melhoria e aperfeiçoamento do material. Entretanto, ressaltam-se as dificuldades de se obter a participação destes, tendo em vista que muitos profissionais se encontraram sobrecarregados com suas jornadas de trabalho, não tendo, portanto, disponibilidade de participar de uma validação como a proposta pelo estudo.

Outras limitações referentes ao estudo foram: a impossibilidade de acesso aos benefícios desta tecnologia pelas gestantes analfabetas, mulheres que não possuem acesso a um smartphone, deficientes sensoriais e intelectuais ou residentes em áreas de difícil acesso à internet. Outra limitação percebida foi a do aplicativo ser disponível apenas para telefones smartphones, dificultando muitas vezes o acesso até mesmo dos especialistas, por estes não possuírem celulares compatíveis.

Por fim, ressalva-se que as sugestões propostas foram acatadas, enriquecendo a produção do aplicativo. $\mathrm{O}$ processo de adaptação da tecnologia às sugestões dos especialistas é uma etapa essencial para tornála mais completa, com maior rigor e eficácia.

\section{CONCLUSÃO}

O aplicativo GestHiper foi considerado válido após o processo de validação feito com os juízes e a consulta a representantes do público-alvo.

Conclui-se que as informações contidas no aplicativo móvel contribuirão de forma significante para a aquisição de conhecimento por parte das gestantes que dele se utilizar, permitindo também o monitoramento mais efetivo da sua pressão https://doi.org/10.31011/reaid-2021-v.95-n.35-art.1186 Rev Enferm Atual In Derme v. 95, n. 35, 2021 e-021124 
arterial, através das ferramentas que o aplicativo disponibiliza.

Diante das limitações mencionadas anteriormente, uma sugestão para futuros trabalhos nessa temática seria o desenvolvimento de um material com a presença de outros recursos audiovisuais, como a presença de áudio em todos os textos. Também seria interessante a elaboração de um material em Braille do conteúdo do aplicativo.

Acredita-se que o aplicativo móvel será um grande estímulo para uma maior produção de trabalhos nesta área, podendo-se assim aproveitar a imersão populacional nas tecnologias para promover o uso de ferramentas voltadas a saúde e ao autocuidado dos indivíduos.

Não há conflito de interesse na publicação do artigo.

\section{REFERÊNCIAS}

1. Maldonado MTP. Psicologia da gravidez, parto e puerpério. Petrópolis (RJ): Vozes; 1976.

2. Sanine PR, Venancio SI, Silva FLG, Aratani N, Moita MLG, Tanaka OY. Atenção ao pré-natal de gestantes de risco e fatores associados no Município de São Paulo, Brasil. Cad. Saúde Pública [Internet]. 2019 [acesso em 28 Jan 2020]; 35(10): e00103118. Disponível em: https://www.scielosp.org/article/csp/2019.v35 $\underline{\text { n10/e00103118/ }}$

3. Cunha AC, Lacerda JT, Alcauza MTR, Natal S. Avaliação da atenção ao pré-natal na
Atenção Básica no Brasil. Rev. Bras. Saúde Mater Infant [Internet]. 2019 [acesso em 28 Jan 2020]; 19( 2 ): 447-58. Disponível em: https://www.scielo.br/j/rbsmi/a/j9DVWHCJV YZCD46FPxwb4Wk/?lang=pt

4. Oliveira JCS, Santos AAP, Santos WB, Santos JAM, Teixeira LM, Rodrigues RPGTO, Acioli DMN. Maternal mortality: epidemiological profile of a state of northeast Brazil. Research, Society and Development [Internet]. 2020 [cited 2021 Jun 10]; 9(10): e9859109310. Available from: https://rsdjournal.org/index.php/rsd/article/vie w/9310

5. Fiorio TA, Pereira TM, Righi MG, Vieira AP, Follador FAC, Wendt GW, Farreto LED. Doença hipertensiva específica da gestação: prevalência e fatores associados. Brazilian Journal of Development [Internet]. 2020 [acesso em 10 jun 2021]; 6(6): 35921-35934. Disponível

em: https://www.brazilianjournals.com/index.php/ BRJD/article/view/11399

6. Falkembach GAM. Concepção e Desenvolvimento de Material Educativo Digital. RENOTE - Revista de Novas Tecnologias na Educação. [Internet]. 2005 [cited 2016 Oct]; 3(1): 01-14. Available from: http://seer.ufrgs.br/renote/article/view/13742/ 7970

7. Bezerra MAA, Bandeira ENS, Diniz SB, Simões Neto JC, Pereira CCB, Lourenço CO, et al. Construção e Validação de Tecnologia Educativa para Promoção do Processo Ensino-Aprendizagem. Rev. Mult. Psic [Internet]. 2019 [acesso em 28 Jan 2020]; 13 (47): 465-77. Disponível em: https://idonline.emnuvens.com.br/id/article/vi $\underline{\text { ew/2019/3135 }}$

8. Pasquali L. Psicometria: teoria e aplicações. Brasília (BR): UnB; 1997.

9. Pereira FGF, Rocha DJL, Melo GAA, Jaques RMPL, Formiga LMF. Building and validating a digital application for the teaching of surgical instrumentation. Cogitare enferm [Internet]. 2019 [cited 2020 feb 10]; 
24: e58334. Available from: https://revistas.ufpr.br/cogitare/article/view/5 8334

10. Pressman RS. Engenharia de software. 7. ed. Brasil (BR): McGraw Hill Brasil; 2011.

11. Polit DF, Beck CT. Fundamentos da pesquisa em enfermagem: avaliação de evidências para a prática de enfermagem. 7 . ed. Porto Alegre (RS): Artes Médicas; 2011.

12. Lynn MR. Determination and quantification of contente validity. Nurs. Res. [Internet]. 1986 [cited 2017 Dec 15]; 35(6): 382-85. Available from: https://journals.lww.com/nursingresearchonli ne/Citation/1986/11000/Determination_and Quantification_Of_Content.17.aspx

13. MacKinnon GE, Brittain EL. Mobile Health Technologies in Cardiopulmonary Disease. Chest [Internet]. 2020[cited 2021 Jun 10]; 157(3): 654-664. Available from: https://www.ncbi.nlm.nih.gov/pmc/articles/P $\underline{\mathrm{MC} 7078586}$

14. Al-Ratrout $\mathrm{S}$, Tarawneh $\mathrm{OH}$, HusniAltarawneh M, Altarawneh MY. Mobile application development methodologies adopted in omani market: a comparative study. Int $\mathbf{J}$ Eng Res Appl [Internet]. 2019 [cited 2020 feb 15]; 10 (2): 13-22. Available from: https://papers.ssrn.com/sol3/papers.cfm?abstr act_id=3376391

15. - Rasburn M, Livingstone H, Scott S. Strengthening patient outcome evidence in health technology assessment: A coproduction approach. International Journal of Technology Assessment in Health Care [Internet]. 2021 [cited 2021 Jun. 11]; 37(1): e12.Available from: https://www.cambridge.org/core/journals/inte rnational-journal-of-technology-assessmentin-health-care/article/strengthening-patientoutcome-evidence-in-health-technologyassessment-a-coproduction approach/B6B15E9A05F02E820270BCB40A $\underline{184898}$
16. Sales RO, Dilts LM, Silva RM, Brasil CCP, Vasconcelos Filho JE. Desenvolvimento e avaliação de um aplicativo para o controle da sífilis em gestantes. Rev. Bras. Enferm. [Internet]. 2019 [acesso em 02 Mar 2020]; 72(5): 132632. Disponível em: https://www.scielo.br/j/reben/a/HkXRk7L4M 7BW4mWC9RbscxM/abstract/?lang=pt

17. Pereira FGF, Rocha DJL, Melo GAA, Jaques RMPL, Formiga LMF. Construção e validação de aplicativo digital para ensino de instrumentação cirúrgica. Cogitare enferm [Internet]. 2019 [acesso em 11 Jun 2021]; 24: e58334. Disponível em: https://revistas.ufpr.br/cogitare/article/view/5 $\underline{8334 / \mathrm{pdf}}$

18. Barros Júnior FS, Barradas Júnior AR, Lima JC, Aires IO, Rocha CB, Rêgo Neta $\mathrm{MM}$, et al. Perfil antropométrico de gestantes internas com diagnóstico de pré-eclâmpsia grave. J. nurs. health. [Internet]. 2019 [acesso em 05 Mar 2020]; 9(3):e199309. Disponível em:

https://periodicos.ufpel.edu.br/ojs2/index.php/ enfermagem/article/view/16812

Submissão: 2021-07-30

Aprovado: 2021-08-22 